\title{
Neutron Detection at the Extremes of Sensitivity in the Cosmic Environment
}

\author{
Chris Benson, Malcolm J. Joyce, Barry O'Connell, and Jon Silvie
}

\begin{abstract}
This paper compares and contrasts the use of MOSFET dosimeters and extended rem counters for neutron detection in cosmic radiation fields. These technologies have been exposed to a reference field and data are presented that demonstrate the contrasting sensitivities of these approaches. The MOSFETs are observed to be insensitive to dose levels associated with cosmic fields whilst the extended rem counters give a good response, demonstrating that copper has potential as a replacement for lead in these systems.
\end{abstract}

\section{INTRODUCTION}

$\mathbf{T}$ HE EARTH is constantly being bombarded by cosmic radiation of both galactic and solar origin. These particles range in energy up to $10^{20} \mathrm{eV}$ (galactic) with the majority being very high-energy protons [1], [2]. The surface of the earth is shielded from these high-energy particles by approximately $1000 \mathrm{~g} . \mathrm{cm}^{-2}$ of air and, as such, this radiation contributes less than $10 \%$ to the total radiation exposure at sea level [3]. However, this radiation interacts with nitrogen, oxygen and other constituent atoms in the Earth's atmosphere to produce a variety of secondary particles which are often of greater relative biological effectiveness than the primary particles, such as neutrons, for example. This phenomenon results in a maximum intensity at an altitude of approximately $20 \mathrm{~km}$, known as the Pfotzer maximum. The cosmic neutron component is assuming greater importance at these altitudes due to the previous underestimation of its relative biological effectiveness [4], [5], the revised recommendations of the International Commission on Radiological Protection (ICRP) [6], [7] and the European Union requirements for the radiation protection of aircraft crew [8]. These aspects are of specific concern to the air transport industry since longer periods of time are being spent in aircraft at ever increasing altitudes. The use of air travel has been steadily growing for the past 30 years and is currently experiencing $5 \%$ growth per year; it is expected that in 20 years time, three times as much traffic will be using air travel [9]. Consequently, aircraft may spend longer periods of time at

Manuscript received July 25, 2000. This work was supported in part by the UK Engineering and Physical Sciences Research Council and by BAe Systems, Operations, Marine, UK.

C. Benson and M. J. Joyce are with the Department of Engineering, Lancaster University, LA1 4YR UK (e-mail: \{c.benson; m.joyce\} @lancaster.ac.uk).

B. O'Connell, was with the National Microelectronics Research Centre (NMRC), University College, Lee Maltings, Cork, Ireland. He is now with the National Semiconductor Corporation, Santa Clara, CA 95052 USA (e-mail: Barry.O’Connell@nsc.com).

J. Silvie is with Instrument Development Services, BAe Systems, Operations, Marine, Barrow-in-Furness, Cumbria, LA14 1AF UK (e-mail: jonathon.silvie@baesystems.com).

Publisher Item Identifier S 0018-9499(00)11181-5. altitudes associated with increased radiation dose, a specific concern for the very-frequent long-haul business traveller. It is clear, therefore, that improved radiation monitoring of the cosmic environment is of considerable technological interest, from both academic and commercial perspectives.

Several dosimetry techniques have been recommended [6] for use in the cosmic environment associated with commercial aviation. These include; passive personal devices, such as thermoluminescence dosemeters and etched-track devices; active personal devices, including solid-state devices and there is also a complementary variety of approaches to the improved characterization of the radiation field. Radiation monitoring on-board aircraft is well established for research purposes [10]-[15] and monitoring on-board commercial supersonic aircraft has been ongoing successfully for more than 20 years [16], [17]. However, the equipment used in the latter is antiquated in comparison with current design approaches.

In this paper, we report on a selection of new measurements concerning electronic dosimetry systems in the cosmic neutron environment associated with high-altitude air travel. These systems span the sensitivity extremes possible with modern dosimetry approaches and comprise:

- boron-implanted large-geometry MOSFET devices, supplemented by a variety of hydrogenous layers;

- a pair of polythene-moderated proportional counters augmented by copper/lead shells.

We present the first measurements of the response of highsensitivity MOSFETs to a cosmic neutron reference field, which is compared to the more familiar response of two proportional counter designs.

\section{BACKGROUND}

The sensitivity of MOS transistors to radiation is well-established and is manifest as the change in threshold $\left(V_{t}\right)$, or flatband voltage $\left(V_{f b}\right)$, in response to radiation exposure [18]. In the past, MOS transistors have been referred to as the Space-Charge transducer, the MOS dosimeter [19] and, most often, as the RadFET (RADiation Field Effect Transistor) [20]. The mechanism by which MOS transistors respond to radiation is through charge- and interface-state build-up in the gate oxide $\left(\mathrm{SiO}_{2}\right)$. Devices based on the pMOS process exhibit greater change in threshold or flatband voltage characteristics, as the contribution of both interface states and charge build-up is in the same, negative direction. It has been observed that nMOS devices can exhibit rebound where the two contributing factors cancel each other out with either no overall change, or with a positive change (increase) being observed [21]. 
In previous studies, MOS transistors have been reported as exhibiting a threshold voltage shift when exposed to tens of $\mu \mathrm{Sv}$ [22] in tests that have predominantly used $\gamma$-radiation. Greatly improved $\gamma$-ray sensitivity has been reported on MOS devices since these measurements, due to process optimization [23], temperature compensation [24] and multidevice stacking [25], [26].

The response of MOS devices to mixed fields has been studied comparatively little, with reported neutron sensitivities for bare devices of $65 \mathrm{mV} / \mathrm{Gy}(\mathrm{Si})$ [27]. Indeed, the neutron insensitivity has often been the basis for the use of these devices in discriminating the $\gamma$-component of a mixed field [28]. More recently, hydrogenous material has been used to sensitize MOS devices to neutrons [29], heralding the devices as suitable solid-state bases for mixed-field personal dose measurement for military personnel. Complementary measurements have been undertaken for medical applications that compare the potential of MOSFETs for combined macro- and micro-dosimetry, exploiting water to produce secondary protons [30]. We are not aware of any previous attempts to measure neutron dose with the high-sensitivity MOSFETs used in this work, nor are we aware of any attempts to assess the rationale of using hydrogenous converters to sensitize any MOSFET devices to the cosmic environment where the sensitivity of these is likely to be different. Conversely, neutron field characterization has long been undertaken with systems based on proportional counters [31] in combination with hydrogenous moderators. The energy response of these instruments can be extended for the cosmic environment by the incorporation of high- $Z$ materials into the moderator [32], [35] to promote $(n x n)$ reactions of fast neutrons. Bonner sphere sets have been developed and modeled on this basis for the spectroscopy of cosmic fields [36]. Thus, design optimization remains the focus of much research regarding the inevitable compromise of detection sensitivity, physical size/mass and manufacturing simplicity demanded by commercial instruments based on this technology.

\section{EXPERIMENTAL}

In total, eighteen MOS devices were irradiated at the CERN Reference Field Facility (CERF). Seven of these devices were ion-implanted with boron, in order to adjust the threshold voltage therefore minimizing power source requirements and, further, to optimize radiation sensitivity. This latter optimization is due to an increase in hole-traps introduced to the oxide by the ion-implantation process. A number of the devices tested had the surface of the silicon wafer covered with layers of polyethene of different thicknesses to promote $(n, p)$ reactions with the high-energy neutrons and thus enhance the device response to the neutron component of the radiation field. The thickness of the layers applied was based upon stopping-power data for protons in polyethene [37]. Layers of $1-3 \mathrm{~mm}$ were chosen to suit the proton energies expected following elastic interactions with the very high-energy neutrons involved [38].

The MOS devices used in this work were fabricated by the National Microelectronics Research Centre (NMRC) in Cork, Ireland. The NMRC devices are pMOS transistors with a thermal $400 \mathrm{~nm}$ silicon dioxide layer grown at $1000^{\circ} \mathrm{C}$
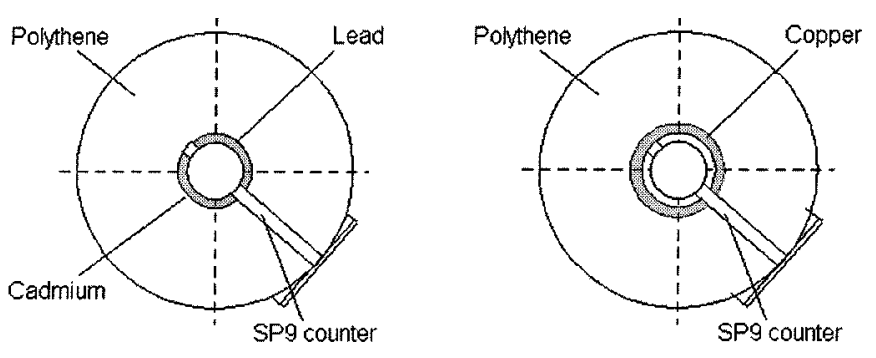

Fig. 1. Cross-sectional view of both polythene-moderated proportional counters.

[39]. The devices tested have a gate geometry of $300 / 50 \mu \mathrm{m}$ (width/length). The processing of these devices has been optimized to provide radiation softness and so represents the best case for a radiation dosimeter or, alternatively, the worst case for an operational MOS device used in cosmic fields as an avionic component.

The devices were all irradiated with a gate bias of $+5 \mathrm{~V}$ and all other connections grounded; this creates a $1.25 \mathrm{MV} / \mathrm{cm}$ field across the gate oxide. This field reduces hole-electron recombination in $\mathrm{SiO}_{2}$ and enhances hole-trapping within the oxide, thereby providing a greater threshold voltage shift. The threshold voltage of these devices was recorded pre-, mid- and post-irradiation in the user area at CERF. The time incurred between exposure and reading of the devices was a maximum of a few minutes.

The extended rem counter system, also tested at CERF, consisted of two, $208 \mathrm{~mm}$ diameter polyethylene moderating spheres with a central shell of either i) an inner layer of lead surrounded by a shell of cadmium or ii) an inner layer of copper. Both of these designs were undertaken to exploit the $(n, x n)$ reaction of fast neutrons. Copper was chosen as an alternative to lead because it is easier to machine and generally less hazardous. In this case, the cadmium surround was removed in order to adjust the fenestration ratio which was judged to be potentially detrimental due to the exclusion of thermalized cosmic neutrons from the central counter. The central detectors in these effectively-modified Leake [31] arrangements are SP9 ${ }^{3} \mathrm{He}$ counters at $2 \mathrm{~atm}$. pressure. The instruments have integrated electronic support, comprising high-voltage supply and pre-amplification. Data were taken, via bespoke DC-2 data-logger devices developed and supplied by John Caunt Scientific, Oxford, UK. Data were downloaded and processed via RS-232 interface to a PC after the tests. The cross section of both detectors is given in Fig. 1.

Since 1993, high-energy stray radiation fields have been available at CERN [40], Geneva. The Reference Field Facility, installed at the Super Proton Synchrotron (SPS) accelerator, can provide reference fields similar to those found at flying altitudes of $10-15 \mathrm{~km}$. The reference field is created by bombarding a copper target, $7 \mathrm{~cm}$ in diameter and $50 \mathrm{~cm}$ in length with a beam of positive or negative hadrons with a momentum of either $120 \mathrm{GeV} / \mathrm{c}$ or $205 \mathrm{GeV} / \mathrm{c}$. The reference field is located at $90^{\circ}$ to the beam of hadrons, behind an $80 \mathrm{~cm}$ shield of concrete. The areal mass similarity between the atmospheric air layer above $10-15 \mathrm{~km}$ and the concrete shield reproduces the cosmic neutron energy distribution, as shown by Fig. 2. The 


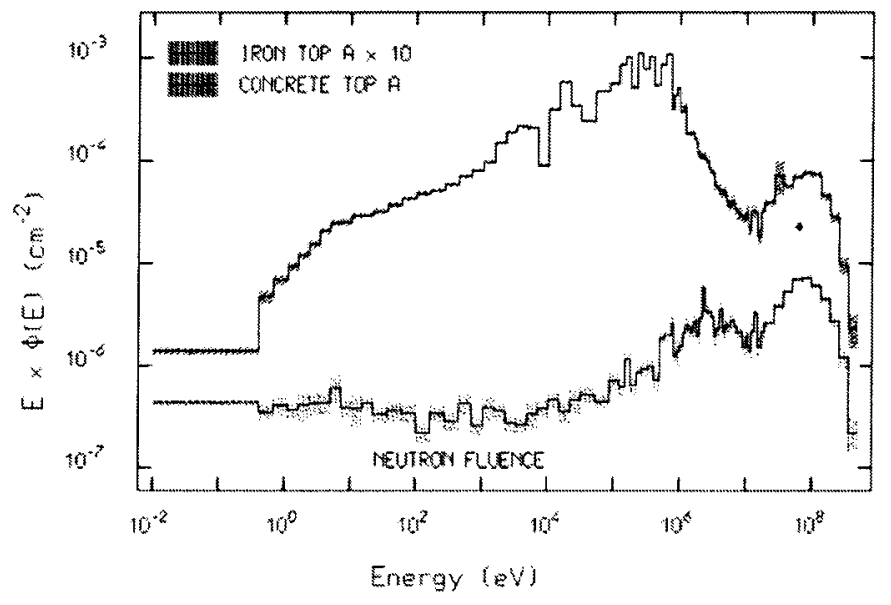

Fig. 2. Neutron energy distribution of fluence for CERN reference field, calculated with Monte Carlo code FLUKA. Reproduced with kind permission from [41].

TABLE I

Dose Received By Devices Over the 24-Hour EXPerimental PeRIOD

\begin{tabular}{l|l|l}
\hline Detector & $\mathrm{Pb}$ & $\mathrm{Cu}$ \\
\hline $\begin{array}{l}\text { Sensitivity } \\
\text { (counts/nSv) }\end{array}$ & $0.32 \pm 0.03$ & $2.04 \pm 0.19$ \\
\hline
\end{tabular}

SPS beam is pulsed exactly 250 times per hour. This creates a near-uniform radiation field over a $4 \mathrm{~m}^{2}$ area above the concrete shield, which is sub-divided into sixteen numbered sub-areas, $0.25 \mathrm{~m}^{2}$ in size. A precision ion chamber (PIC) present at the facility is used to count the number of particles per pulse, which can be converted to dose based on a known calibration, according to both ICRP 21 and 60 [42], [43].

\section{RESULTS}

The MOSFETs and the extended rem counters were placed in the field for three, eight-hour periods. The total dose received by each of the locations over the twenty-four hours is detailed in Table I. These data were obtained from reference values measured previously with the HANDI TEPC [43] for each of the sub-areas.

The threshold voltages $\left(V_{t}\right)$ before, during and after irradiation for the nonimplanted MOSFETs are shown in Fig. 3 and for the implanted devices in Fig. 4. The count response of the spherical detectors is compared in Fig. 5, in terms of cumulative counts versus time for an eight-hour shift. There are a few flat spots in these data due to occasional beam down-time of the beam during this particular shift. In Fig. 6, the time scale is expanded to show the detector response to the short-period pulsed character of the field at CERF. From these data, the count sensitivity has been determined, as given in Table II.

\section{DISCUSSION}

The data presented in Figs. 3 and 4 demonstrate that a change in threshold voltage has not been observed for the MOS devices. It is however noted that there appears to be slight inconsistency between the readings for MOSFETs 58-61, which is not exhibited by the ion-implanted devices, for which

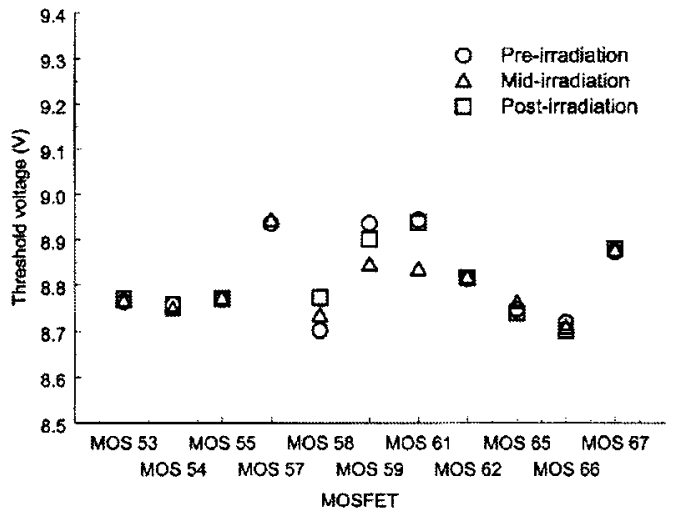

Fig. 3. Threshold voltage measurements for nonimplanted MOSFETs.

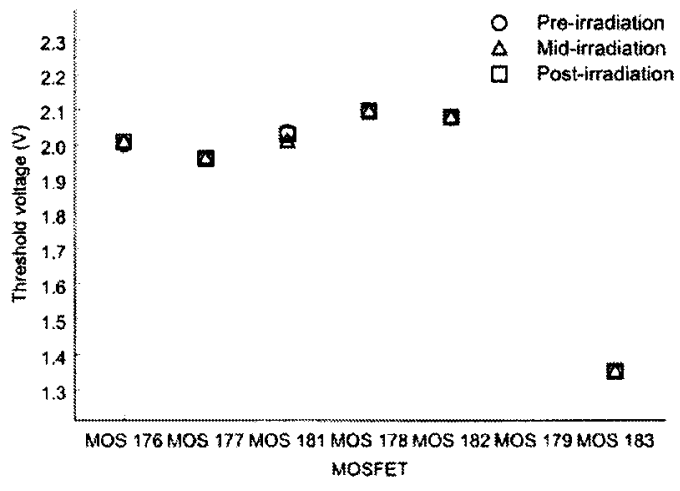

Fig. 4. Threshold voltage measurements for ion implanted MOSFETs.

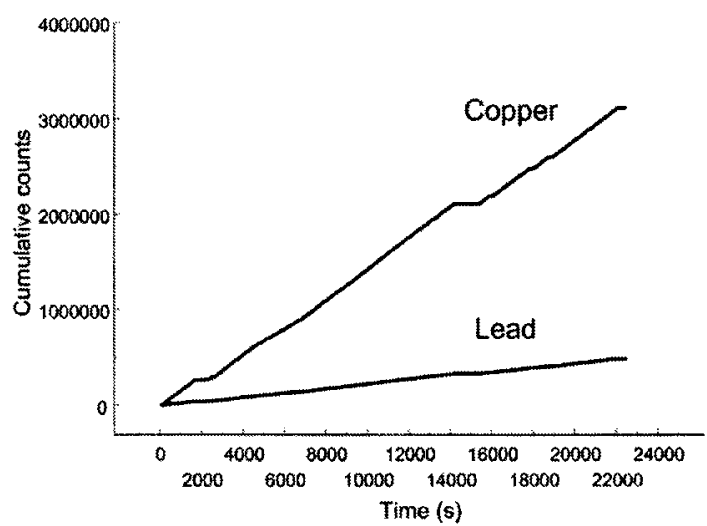

Fig. 5. Response of proportional counters during 8-h exposure.

further investigation is required. Some of the devices, such as MOS176, MOS177 and MOS181, exhibit trends that are consistent with the small threshold change expected. However, other devices contradict this observation and the data are therefore inconclusive.

It is clearly ambitious to expect a large MOSFET response for such low doses whilst previous investigations of neutron fields with MOSFET dosimeters [28], [29] have often used much higher-dose, lower-energy sources than those attempted in this work. In this respect, fading might be expected to be comparable with any low-level response. Fading of the radiation response in MOSFETs is well-known to be smallest for devices in which the oxide is thickest and decreases with increase in gate voltage [44]-[46]. The MOSFETs used in this 


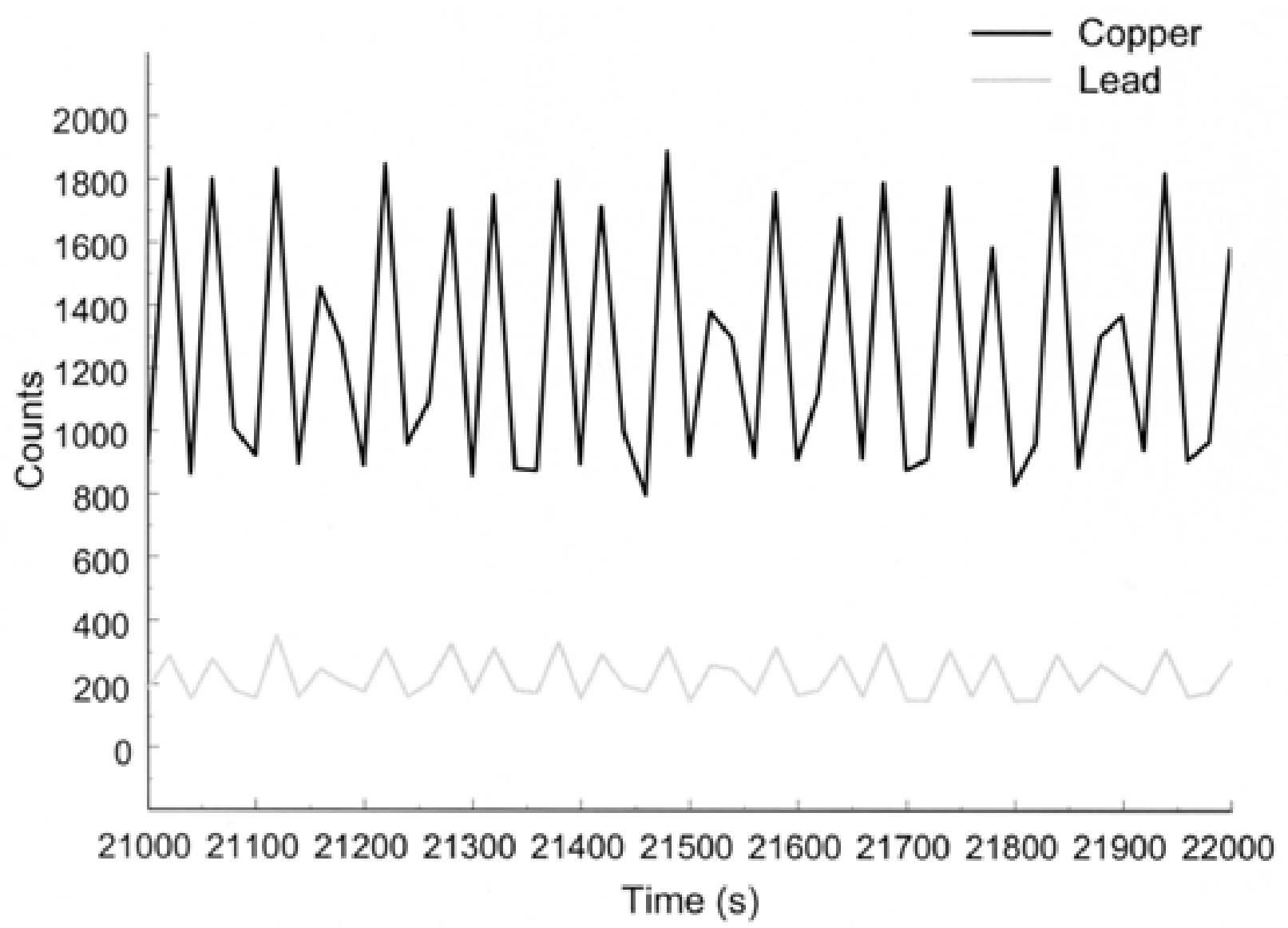

Fig. 6. Response of proportional counters over a short duration.

TABLE II

COUNT SENSITIVITY FOR THE EXTENDED REM COUNTERS

\begin{tabular}{c|c|c|c|c|c|c}
\hline & \multicolumn{2}{|c|}{ MOSFETS } & \multicolumn{2}{c|}{ Pb Detector } & \multicolumn{2}{c}{ Cu Detector } \\
\hline \multirow{2}{*}{$\begin{array}{c}\text { Total Dose } \\
(\mathrm{mSv})\end{array}$} & ICRP 21 & ICRP 60 & ICRP 21 & ICRP 60 & ICRP 21 & ICRP 60 \\
\cline { 2 - 7 } & $5.50+/-0.60$ & $6.35+/-0.55$ & $5.38+1-0.54$ & $6.17+1-0.47$ & $5.34+/-0.6$ & $6.08+1-0.56$ \\
\hline
\end{tabular}

work were of average thickness and the gate voltage used is typical of that used for similar studies of higher-dose $\gamma$-ray environments. However, the duration over which annealing is often observed is much longer (several hours) than the time delay incurred between exposure and reading in this work, especially at ambient temperatures. The fading phenomenon is due to the conflicting effect of increases in interface trap density and decreases in gate-oxide charge density [47], [48]. These often cancel each other in the early stages of annealing, suggesting minimal fading of the response over the time-scales and ambient temperatures involved in this work.

Device sensitivity for the MOSFETs used in this paper is in the $100 \mathrm{mV} / \mathrm{Sv}$ range for $\gamma$-radiation. This indicates a measurement requirement of $\mu \mathrm{V}$ resolution for the doses administered in this research. This is feasible with the equipment used for current measurement but is smaller than the fluctuations caused by drift. Compensation of the temperature-based component of this drift is unlikely to result in the large increase in signal-to-noise ratio necessary to decipher a small effect. A much more attractive route is to increase the gate bias and employ the on-chip multi-MOSFET stacking approach which has been shown to increase sensitivity by factors in excess of two hundred [25], [26], [39] over that of a single device.

The data for the spherical detectors indicate that copper has potential as a replacement for lead as the high- $Z(n, x n)$ converter. The data in this work exhibit a greater sensitivity for the copper-based design. However, it must be emphasized that this design was tested without the cadmium thermal shield and such increased sensitivity could be indicative of the characteristic thermal over-response to which this type of detector is often prone. This is especially relevant because of the large thermal component of the CERF field above the concrete shield. To determine whether this is the case, a complete energy response study is necessary as has been demonstrated comprehensively for the lead design [34]. Greater insight into the dose-response characteristic of this detector design is possible via Monte Carlo modeling in order to optimize the detector dimensions.

\section{CONCLUSION}

In this paper, we have reported the first attempt at cosmic neutron detection in large-geometry MOSFET devices; both 
implanted/nonimplanted which have been sensitized by a selection of hydrogenous layers. These data have been contrasted to the responses of two designs of the extended-range neutron rem counter. In summary, the MOSFET devices have been shown to be too insensitive for cosmic neutron dosimetry in their present form despite the enhancements described above. More research is necessary to investigate alternative methods of sensitivity increase in these devices.

The measurements with the extended rem counters demonstrate a good count response, consistent with the considerable prior-art in this aspect of the study. They demonstrate that copper has potential as a replacement for lead with the considerable benefits this has for detector manufacturing practices. However, more research is necessary to elucidate the energy response and degree of likely over-response in the thermal region for these counters.

\section{ACKNOWLEDGMENT}

The authors would like to thank B. Mason of Centronic Ltd. for the many useful discussions and for the preparation and manufacture of the polyethylene spheres and SP9 counters used in this program. They would also like to thank Drs. D. Bartlett and R. Tanner, of the National Radiological Protection Board (NRPB), and Dr. D. Thomas of the National Physical Laboratory for their help and advice. They would also like to thank A. Mitaroff and Dr. M. Silari for their assistance at CERN.

\section{REFERENCES}

[1] W. Heinrich, S. Roesler, and H. Schraube, "Physics of cosmic radiation fields," Rad. Prot. Dos., vol. 86, no. 4, pp. 253-258, 1999.

[2] G. Reitz, "Radiation environment in the stratosphere," Rad. Prot. Dos. vol. 48, no. 1, pp. 5-20, 1991.

[3] H. G. Paretzke and W. Heinrich, "Radiation exposure and radiation risk in civil aircraft," Rad. Prot. Dos., vol. 48, no. 1, pp. 33-40, 1991.

[4] R. J. Donahue and A. Fasso, "Radioactivity and radiation protection," European Journal of Physics, vol. 3, 1998.

[5] R. J. Barish, "Health physics concerns in commercial aviation," Health Physics, vol. 59, no. 2, pp. 199-204, 1990.

[6] D. T. Bartlett, "Dosimetry methods for the measurement of the radiation exposure of civil air crew," Rad. Prot. Dos., vol. 48, no. 1, pp. 93-100, 1993.

[7] ICRP. 1990 Recommendations of the International Commission on Radiological Protection: Pergamon Press, 1991.

[8] D. T. Bartlett, "Radiation protection concepts and quantities for the occupational exposure to cosmic radiation," Rad. Prot. Dos., vol. 86, no. 4, pp. 263-268, 1999.

[9] C. J. Hume, "Cosmic radiation — an aircraft manufacturer's view," Rad. Prot. Dos., vol. 86, no. 4, pp. 335-336, 1999.

[10] L. Tommasino, "In-flight measurements of radiation fields and doses," Rad. Prot. Dos., vol. 86, no. 4, pp. 297-301, 1999.

[11] R. H. Thomas, "Ionising radiation exposure measurements at commercial jet aircraft altitudes," Rad. Prot. Dos., vol. 48, no. 1, pp. 51-57, 1993.

[12] Y. A. Akatov, "Some results of dose measurements along civil airways in the USSR," Rad. Prot. Dos., vol. 48, no. 1, pp. 59-63, 1993.

[13] D. Regulla and J. David, "Measurements of cosmic radiation on board Lufthansa aircraft on the major intercontinental flight routes," Rad. Prot. Dos., vol. 48, no. 1, pp. 65-72, 1993.

[14] F. Spurny, O. Obraz, F. Pernièka, I. Votoèkova, K. Turek, V. D. Nguyen, O. Vojtisek, V. Starostová, and O. Kol¡n, "Dosimetric characteristics of radiation fields on board czechoslavak airlines aircraft as measured with different active and passive detectors," Rad. Prot. Dos., vol. 48, no. 1 , pp. 73-77, 1993.

[15] C. Montagne, J. P. Donne, D. Pelcot, V. D. Nguyen, P. Bouisset, and G Kerlau, "In-flight radiation measurements aboard french airlines," Rad. Prot. Dos., vol. 48, no. 1, pp. 79-83, 1993.
[16] D. M. Davies, "Cosmic radiation in concorde operations and the impact of new ICRP recommendations on commercial aviation," Rad. Prot. Dos., vol. 48, no. 1, pp. 121-124, 1993.

[17] M. Bagshaw, "Cosmic radiation measurements in airline service," Rad. Prot. Dos., vol. 86, no. 4, pp. 333-334, 1999.

[18] A. Holmes-Siedle, "The space charge dosimeter," Nucl. Inst. Meth., vol. 121, pp. 169-174, 1974.

[19] L. S. August, "Estimating and reducing errors in MOS dosimeters caused by exposure to different radiations," IEEE Trans. Nucl. Sci., vol. 29, pp. 2000-2003, 1982.

[20] R. C. Hughes, "Theory of response of radiation sensing field-effect transistors," J. Appl. Phys., vol. 58, no. 3, pp. 1375-1379, 1985.

[21] J. R. Schwank, P. S. Winokur, P. J. McWhorter, and F. W. Sexton, "Physical mechanisms contributing to device rebound," IEEE Trans. Nucl. Sci., vol. 31, pp. 1434-1438, December 1984.

[22] C. M. Conneely, "Optimization strategies for PMOS dosimeters," M.Sc. Thesis, University College Cork, 1997.

[23] B. O'Connell, "Personal communication," unpublished, 1999.

[24] C. Conneely, B. O'Connell, P. Hurley, W. Lane, and L. Adams, "Strategies for millirad sensitivity in PMOS dosimeters," IEEE Trans. Nucl. Sci., vol. 45, pp. 1475-1480, 1998.

[25] A. Kelleher, W. Lane, and L. Adams, "A design solution to increasing the sensitivity of pMOS dosimeters: The stacked RADFET approach," IEEE Trans. Nucl. Sci., vol. 42, pp. 48-51, 1995.

[26] B. O'Connell, A. Kelleher, W. Lane, and L. Adams, "Stacked RADFETs for increased radiation sensitivity," IEEE Trans. Nucl. Sci., vol. 43, pp. 985-990, 1996.

[27] N. G. Blamires and D. H. J. Totterdell, "PMOS dosimeters: Long-term annealing and neutron response," IEEE Trans. Nucl. Sci., vol. 33, no. 6, December 1986.

[28] A. Rosenfeld, V. Khivrich, V. Kuts, M. Tavlet, L. Malfante, and C. Munoz-Ferrada, "Use of Ukrainian semiconductor dosimeters in a CERN particle accelerator field," IEEE Trans. Nucl. Sci., vol. 41, pp. 1009-1013, 1994.

[29] S. Kronenberg and G. J. Brucker, "The use of hydrogenous material for sensitizing pMOS dosimeters to neutrons," IEEE Trans. Nucl. Sci., vol. 42, no. 1, pp. 20-26, 1995.

[30] A. B. Rosenfeld, G. I. Kaplan, M. G. Caroian, B. J. Allen, R. Maughan, M. Yudelev, C. Kora, and J. Coderre, "Simultaneous macro and micro dosimetry with MOSFETs," IEEE Trans. Nucl. Sci., vol. 43, no. 6, pp. 2693-2700, 1996.

[31] J. W. Leake, “An improved spherical dose equivalent neutron detector,' Nucl. Inst. Meth., vol. 63, pp. 329-340, 1968.

[32] C. Birattari, A. Ferrari, C. Nuccetelli, M. Pelliccioni, and M. Silari, "An extended range neutron rem counter," IEEE Trans. Nucl. Sci., vol. A297, pp. 230-257, 1990 .

[33] - "A neutron survey meter with sensitivity extended up to 400 MeV," Rad. Prot. Dos., vol. 44, pp. 193-197, 1992.

[34] - "The extended range neutron rem counter 'LINUS': Overview and latest developments," Rad. Prot. Dos., vol. 76, no. 3, pp. 135-148, 1998

[35] V. Vylet, J. C. Liu, S. H. Rokni, and L. X. Thai, "Measurements of neutron spectra at the Stanford linear accelerator center," Rad. Prot. Dos., vol. 70, no. 1-4, pp. 425-428, 1997.

[36] V. Vylet, "Response matrix of an extended Bonner sphere system," in International Workshop on Neutron Field Spectrometry in Science, Technology and Radiation Protection, Pisa, Italy, June 4-8, 2000.

[37] "Stopping powers and ranges for protons and alpha particles," ICRU, ICRU Report 49, 1993.

[38] J. F. Ziegler, "Personal communication," unpublished, April 2000.

[39] B. O'Connell, C. Conneely, C. McCarthy, J. Doyle, W. Lane, and L. Adams, "Electrical performance and radiation sensitivity of stacked PMOS dosimeters under bulkbias control," IEEE Trans. Nucl. SSci., vol. 45, pp. 2689-2694, December 1998.

[40] U. J. Schrewe, W. G. Alberts, A. V. Alevra, A. Ferrari, T. Otto, and M. Silari, "Calibration problems, calibration procedures and reference fields for dosimetry at flight altitudes," Rad. Prot. Dos., vol. 86, no. 4, pp. 289-295, 1999.

[41] C. Birattari, T. Rancati, A. Ferrari, A. Hoefert, T. Otto, and M. Silari, "Recent results at the CERN-EC high energy reference field facility," in Proc. Of the 3rd Workshop on Shielding Aspects of Accelerators, Targets and Irradiation Facilities, Sendai, Japan, May 12-13, 1997/1998, OECD/NEA, pp. 219-244.

[42] M. Hofert and G. R. Stevenson, "The CERN-CEC high-energy reference field facility,", CERN Report CERN/TIS-RP/94-02/CF, 1994.

[43] E. Nava, T. Otto, and M. Silari, "Reference dose equivalent values for the 1997 CERN-EC runs,", CERN Tech. Memo. CERN/TIS- RP/97-22, 1997. 
[44] A. Kelleher, M. O'Sullivan, J. Ryan, B. O'Neill, and W. Lane, "Development of the radiation sensitivity of PMOS dosimeters," IEEE Trans. Nucl. Sci., vol. 39, pp. 342-346, 1992.

[45] A. Kelleher, N. McDonnell, B. O'Neill, and W. Lane, "The effect of gateoxide process variations on the long-term fading of PMOS dosimeters," Sensors and Actuators, vol. A37-38, pp. 370-374, 1993.

[46] G. Risti, S. Golubovi, and M. Pejovi, "P-channel metal-oxide- semiconductor dosimeter fading dependencies on gate bias and oxide thickness," Appl. Phys. Lett., vol. 66, no. 1, pp. 88-89, 1995.
[47] G. Risti, A. Jaki, and M. Pejovi, "pMOS dosimetric transistors with twolayer gate oxide," Sensors and Actuators, vol. 63, pp. 129-134, 1997.

[48] G. Risti, S. Golubovi, and M. Pejovi, "Sensitivity and fading of pMOS dosimeters with thick gate oxide," Sensors and Actuators, vol. 51, pp. $153-158,1996$. 